\title{
Effect of Preventive Messages Tailored to Family History on Health Behaviors: The Family Healthware Impact Trial
}

\author{
Mack T. Ruffin IV, MD, MPH \\ Donald E. Nease, Jr, MD \\ Ananda Sen, $P b D^{1}$ \\ Wilson D. Pace, $M D^{2,3}$ \\ Catharine Wang, $\mathrm{PbD}, \mathrm{MSc} \mathrm{c}^{4}$ \\ Louise S. Acheson, MD, MS $S^{5,6,7}$ \\ Wendy S. Rubinstein, $M D, P b D^{8,9}$ \\ Suzanne O'Neill, MA, MS, $P b D^{8,10}$ \\ Robert Gramling, $M D, D S c^{11}$ \\ for the Family History Impact Trial \\ (FHITr) Group
}

'Department of Family Medicine, University of Michigan, Ann Arbor, Michigan

${ }^{2}$ Department of Family Medicine, University of Colorado Health Sciences Center, Aurora, Colorado

${ }^{3}$ American Academy of Family Physicians National Research Network, Leawood, Kansas

${ }^{4}$ Boston University School of Public Health, Boston, Massachusetts

${ }^{5}$ Case Western Reserve University, Cleveland, Ohio

${ }^{6}$ University Hospitals Case Medical Center, Cleveland, Ohio

${ }^{7}$ Case Comprehensive Cancer Center, Cleveland, Ohio

${ }^{8}$ NorthShore University Health System, Evanston, Illinois

${ }^{9}$ University of Chicago Pritzker School of Medicine, Chicago, Illinois

${ }^{10}$ Northwestern University, Chicago, Illinois

"University of Rochester, Rochester,

New York



Conflicts of interest: authors report none.

\section{CORRESPONDING AUTHOR}

Mack T. Ruffin IV, MD, MPH 1018 Fuller St

Ann Arbor, MI 48104-1213

mruffin@umich.edu

\begin{abstract}
PURPOSE We wanted to determine the impact of automated family history assessment and tailored messages for coronary heart disease, stroke, diabetes, colorectal, breast, and ovarian cancer on preventive behaviors compared with a standard preventive message.
\end{abstract}

METHODS The study was a cluster-randomized clinical trial that included 41 primary care practices, the majority in the Midwest, using Family Healthware, a self-administered, Web-based tool that assesses familial risk for the diseases and provides personalized risk-tailored messages. Patients in the control group received an age- and sex-specific health message related to lifestyle and screening. Smoking cessation, fruit and vegetable intake, physical activity, aspirin use, blood pressure, and cholesterol and blood glucose screening were assessed at baseline and 6 months after the intervention.

RESULTS Of 4,248 participants, 3,344 (78\%) completed the study. Participants were white $(91 \%)$, female $(70 \%)$, and insured $(97 \%)$, and had a mean age of 50.6 years (range 35-65 years). Intervention participants were more likely to increase daily fruit and vegetable consumption from 5 or fewer servings a day to 5 or more servings a day (OR $=1.29 ; 95 \%$ confidence interval $[\mathrm{Cl}], 1.05-1.58)$ and to increase physical activity (OR $=1.47 ; 95 \% \mathrm{Cl}, 1.08-1.98)$ to 5 to 6 times a week for 30 minutes or more a week. The absolute differences in proportion were $3 \%$ and $4 \%$, respectively. Intervention participants were less likely to move from not having cholesterol screening in the last 5 years to having their cholesterol measured within 5 years $(\mathrm{OR}=0.34 ; 95 \% \mathrm{Cl}, 0.17-0.67)$, with an absolute difference of $15 \%$.

CONCLUSIONS Messages tailored to an individual's familial risk for 6 common diseases modestly increased self-reported physical activity and fruit and vegetable intake but reduced the likelihood of receiving cholesterol screening.

Ann Fam Med 2011;9:3-11. doi:10.1370/afm.1197.

\section{INTRODUCTION}

$\mathrm{F}$ amily history holds promise to target health promotion activities more effectively to individuals and families according to their genomic risk for disease. ${ }^{1,2}$ Family history captures genetic and environmental components of disease, including shared cultures and behaviors. ${ }^{3}$ The efficiency of familial risk stratification was shown in a Utahbased study reporting that $72 \%$ of coronary heart disease clustered in $14 \%$ of families, and $86 \%$ of strokes occurred in $11 \%$ of families. ${ }^{4}$

The extent of family history collected varies widely. ${ }^{5.7}$ Several issues contribute to the underutilization of family history, including time, complexity, and accuracy. ${ }^{5,89}$ Even when family history is collected, physicians may not precisely identify familial risk. ${ }^{10}$ Furthermore, patients may have incomplete knowledge of their family history. ${ }^{11}$ Interventions that efficiently collect family history, automate familial risk stratification, and allow patients 
to confirm and revise their family history with relatives are likely to increase the utility of family history ${ }^{11}$ and the feasibility of family history research in primary care.

Family history allows recommendations for disease prevention and screening to be tailored to one's level of familial predisposition. ${ }^{12,13}$ Currently, however, there is insufficient evidence showing that family history assessment in primary care improves patient outcomes. ${ }^{6,7}$ A recent systematic review of the usefulness of routinely collecting family history in primary care identified 2 uncontrolled before-after studies of adherence to breast cancer screening, with risk defined by family history of breast or ovarian cancer. ${ }^{7}$ In a recent British randomized trial, a computerized tool used by general practitioners for familial cancer risk increased the number and appropriateness of referrals to cancer genetics clinics. ${ }^{14}$ A National Institutes of Health Stateof-the-Science Conference in 2009 concluded that research addressing this area is needed. ${ }^{6}$

To facilitate efficient collection and use of family history for disease prevention, the Centers for Disease Control and Prevention (CDC) created Family Healthware, an interactive online tool that collects and records family history of 6 common diseases (coronary heart disease; stroke; diabetes; and colorectal, breast, and ovarian cancer) and prevention-relevant habits (physical activity, diet, smoking, alcohol use, aspirin use, and screening test use) for each disease. The software immediately analyzes the user input, generates a 3 -tiered risk assessment based on family history for each disease, and provides tailored preventive health messages to the user focused on health behaviors, screening, and family risk category. The Family Healthware report includes a family tree, as well as information about the specific details of family history that increase the respondent's risk. The development and features of Family Healthware are described elsewhere in detail. ${ }^{15}$

The algorithms to classify risk use individuals' reports of their own health history and that of first- and seconddegree relatives, including diseases in maternal or paternal lineages, and age at onset. The algorithm categorizes risks as weak, moderate, and strong according to rules derived from empirical data and validated in epidemio- logic studies. ${ }^{16}$ The labels for each risk category were developed through focus group studies. ${ }^{15}$ Usually, a weak familial risk was assigned if there was no family history or if there was late-onset disease in only 1 second-degree relative. Moderate familial risk was generally assigned if there was only 1 first-degree relative with late-onset disease or 2 second-degree relatives from the same lineage with late-onset disease. Strong familial risk was generally assigned if there was a firstdegree relative with early-onset disease, when multiple relatives were affected, or when a hereditary syndrome was present. Diabetes was also considered in stratifying risk for coronary heart disease. For most common chronic diseases, a moderate familial risk is associated with about a 2 -fold increase in risk over a weak familial risk, and a strong familial risk is associated with about a 3 -fold or greater increase. ${ }^{13}$

In 2003, the CDC selected 3 academic centers to evaluate this new tool: Evanston Northwestern Healthcare (ENH) (now NorthShore University HealthSystem), the University of Michigan (UM), and Case Western Reserve University (CWRU) with the American Academy of Family Physicians' National Research Network (AAFP NRN). The goal of the Family Healthware Impact Trial (FHITr) was to determine whether systematic family history collection, risk assessment, and messages tailored to familial risk influence the adoption of healthy behaviors, recommended health screenings, and family and physician communication related to the 6 diseases.

This analysis examines the effects of Family Healthware-tailored messages compared with generic health messages among participants reporting suboptimal behaviors with respect to smoking, aspirin use, diet (fruit and vegetable intake), physical activity, blood pressure screening, cholesterol screening, and diabetes screening. We hypothesized that patients who completed Family Healthware and received a risk assessment and messages tailored to family health history of the 6 diseases would be more likely to change these lifestyle behaviors or obtain indicated screening when compared with patients receiving a generic preventive health message (Figure 1).

\section{Figure 1. Conceptual model of how tailored messages on family risk status and recommended preventive strategies would result in behavioral changes.}

\begin{tabular}{|c|c|c|c|}
\hline Family Healthware & $\begin{array}{l}\text { Mediators/Moderators } \\
\text { - Perceived risk } \\
\text { - Perceived severity } \\
\text { - Worry } \\
\text { - Perceived control } \\
\text { - Self-efficacy } \\
\text { - Response efficacy }\end{array}$ & $\begin{array}{l}\text { Intermediate Outcomes } \\
\text { - Intention/stage of change } \\
\text { - Communication with family } \\
\text { and health care providers }\end{array}$ & $\begin{array}{l}\text { Behavioral Outcomes } \\
\text { - Diet (fruit/vegetable) } \\
\text { - Physical activity } \\
\text { - Smoking } \\
\text { - Alcohol } \\
\text { - Aspirin use } \\
\text { - Screening adherence }\end{array}$ \\
\hline
\end{tabular}




\section{METHODS}

The study methods are described in detail elsewhere. ${ }^{17}$ Briefly, FHITr used a 2-arm cluster-randomized design, with primary care practices as the unit of randomization. Eligible patients were recruited from 41 primary care practices; they were aged 35 to 65 years and did not have coronary heart disease, stroke, diabetes, or any cancer except skin cancer other than melanoma. Patients were excluded if pregnant or unable to speak or read English. At ENH and CWRU/AAFP NRN, potentially eligible patients with upcoming appointments were sent invitation letters signed by their primary care physicians. At the UM site, invitation letters were sent to the entire panel of eligible patients of participating clinics. Figure 2 displays recruitment and accrual of practices and patients.

Individual protocols were approved in 2004 by institutional review boards (IRBs) at all 3 centers, and a combined protocol was approved by the CDC's IRB. Recruitment took place from November 2005 to March 2007. The allocation to control or intervention was designed to be about 1 to 2 .

In the intervention arm, participants first completed an online baseline questionnaire followed by Family Healthware, which provided personalized familial risk assessment and prevention messages (see Supplemental Appendix

\section{1, available at http://annfammed.}

in org/cgi/content/full/9/1/3/DC1, for a summary of messages). The control group completed the same online baseline questionnaire and received standard prevention messages for the same 6 diseases (Supplemental Appendix 2,

available at http://annfammed.org/

In cgi/content/full/9/1/3/DC1, for the standard health message). Approximately 6 months later, both the intervention and control groups completed a follow-up questionnaire. Then the control group also completed Family Healthware tool.

The baseline survey collected demographics, health status, and use of medical services; screening behaviors and

\section{Figure 2. Consort diagram of practice and participant recruitment.}

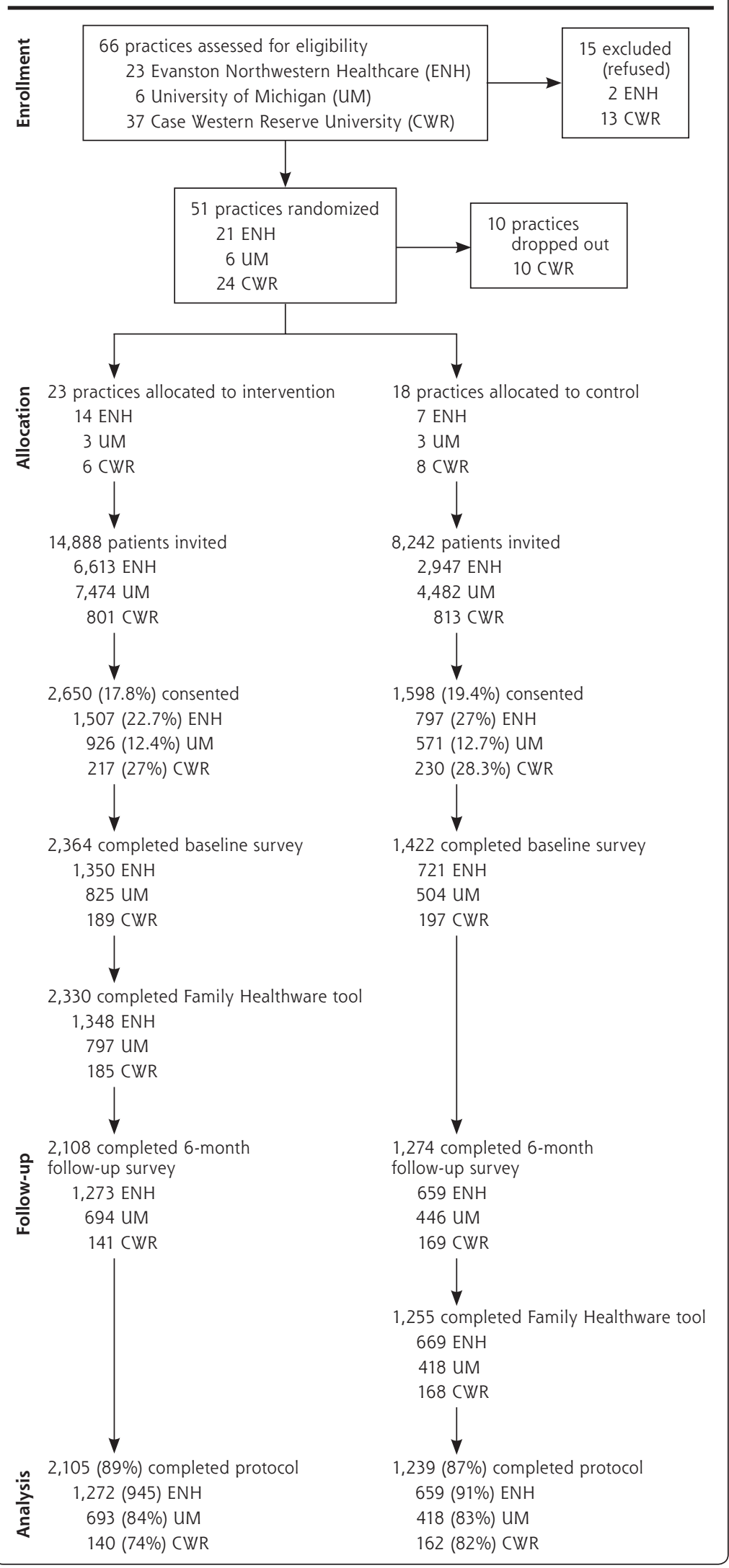


intention to screen; and lifestyle choices, health beliefs, and family history communication patterns (with family members and physicians). The 6-month follow-up survey assessed behavior and health belief changes over time.

Participants viewed their prevention messages on a computer screen after completing the baseline questionnaire. In 2 of the study sites (ENH and CWRU/AAFP NRN), accounting for $67 \%$ of the study population, printed prevention messages were provided to intervention and control groups at a scheduled primary care appointment, with a copy provided to the physician at the patient's request. At the third site (UM), the prevention messages were mailed or e-mailed to the patientwho was asked to bring the messages to the next doctor visit-and copies were given to their physician and placed in the electronic health record. ${ }^{17}$ Physicians at CWRU sites were to complete a questionnaire at the time of each participant's visit. Responses were available for $75 \%$ of intervention group participants, showing that the physician looked at the Family Healthware report during $74 \%$ of these visits (range among physicians, 41\%-100\%).

\section{Outcome Study Measures}

The lifestyle behaviors examined were smoking, fruit and vegetable intake, physical activity, and aspirin use. We measured behavior changes, defined as smoking cessation, increased fruit and vegetable intake to 5 or more servings a day, increased physical activity to 5 to 6 times a week for 30 minutes or more a week, and aspirin use 3 or more days a week. The screening behaviors examined were blood pressure, cholesterol, and blood glucose level measured by a health care professional. The outcomes were evaluated by the percentage of participants obtaining a blood pressure measurement in the last year, a serum cholesterol measurement in last 5 years, and a blood glucose level measurement in the last 2 years. These behaviors were defined as at goal because each behavior's outcome is consistent with common public health messages.

\section{Analysis}

Baseline characteristics were compared across the 2 study arms using a clustered logistic regression model (for categorical variables) or a linear mixed effects model (for continuous variables). The independent variables used in these models were study arm and study site. The clustering effect within practice was accounted for using an exchangeable working correlation structure (for binary logistic or linear models) and an independent correlation structure (for polytomous logistic models, eg, income). We also compared the distribution of participants in the 2 study arms among family history risk categories (weak, moderate, strong) for the 6 diseases by means of an ordinal logistic regression model, with the risk category as outcome and study arm and site as the independent variables. An independence working correlation structure was used to adjust for the clustering effect caused by practice. The distribution of lifestyle and screening behaviors at baseline across study arm, sex, and age was also examined using similar logistic regression models that simultaneously controlled for all these variables. These subgroup analyses were defined a priori.

We compared intervention and control groups by measuring the proportion of study participants moving from not at goal for each lifestyle or screening behavior at baseline to at goal at 6 months. Using binary logistic regression for each behavior outcome, we adjusted for age, body mass index, sex, study site, smoking status (current vs not current), and sum of family history for the 6 diseases not scored as weak at baseline to examine the impact of the intervention compared with the control group. All models were adjusted for practice clustering as described above.

Recruitment season was unevenly distributed between the 2 study arms (Table 1 ), because interven-

\section{Table 1. Demographics of Study Participants} by Study Arm at Baseline

\begin{tabular}{|c|c|c|}
\hline Characteristics & $\begin{array}{c}\text { Intervention } \\
\text { Arm } \\
(n=2,364) \\
\text { No. }(\%)\end{array}$ & $\begin{array}{c}\text { Control } \\
\text { Arm } \\
(n=1,422) \\
\text { No. }(\%)\end{array}$ \\
\hline Sex, female & $1,676(71)$ & $962(68)$ \\
\hline Age, mean (SD), y & $50.3(8.4)$ & $51.1(8.0)$ \\
\hline Hispanic or Latino & $58(2)$ & $29(2)$ \\
\hline \multicolumn{3}{|l|}{ Race } \\
\hline White & $2,134(90)$ & $1,320(93)$ \\
\hline Black or African American & $87(4)$ & $35(3)$ \\
\hline Asian & $70(3)$ & $31(2)$ \\
\hline Other & $42(2)$ & $20(1)$ \\
\hline More than 1 race & $31(1)$ & $16(1)$ \\
\hline \multicolumn{3}{|l|}{ Marital status } \\
\hline Single, never married & $203(9)$ & $96(7)$ \\
\hline Married or living with partner & $1,857(79)$ & $1,135(80)$ \\
\hline Separated or divorced & $260(11)$ & $160(12)$ \\
\hline Widowed & $44(2)$ & $31(2)$ \\
\hline \multicolumn{3}{|l|}{ Season study started } \\
\hline January-April & $1,179(50)$ & $411(29)$ \\
\hline May-August & $704(30)$ & $375(26)$ \\
\hline September-December & $481(20)$ & $636(45)$ \\
\hline \multicolumn{3}{|l|}{ Annual household income ${ }^{a}$} \\
\hline Less than $\$ 25,000$ & $91(4)$ & $41(3)$ \\
\hline$\$ 25,001$ to $\$ 35,000$ & $102(5)$ & $45(4)$ \\
\hline$\$ 35,001$ to $\$ 50,000$ & $218(11)$ & $106(8)$ \\
\hline$\$ 50,001$ to $\$ 75,000$ & $402(19)$ & $228(18)$ \\
\hline More than $\$ 75,000$ & $1,262(61)$ & $834(66)$ \\
\hline Currently has health insurance & $2,276(96)$ & $1,380(97)$ \\
\hline \multicolumn{3}{|c|}{$\begin{array}{l}\text { Note: After adjusting for practice clustering and site differences, the only statis- } \\
\text { tically significant difference between the study arms exists for starting season } \\
(P=.003) \text {. }\end{array}$} \\
\hline \multicolumn{3}{|c|}{ a Income for $12 \%$ was not reported in either group. } \\
\hline
\end{tabular}


tion arm accrual had to be suspended several times while resolving technical problems with Family Healthware. Because small seasonal variations have been found in daily caloric intake, diet composition, and physical activity, ${ }_{1}^{18}$ regression models involving fruit and vegetable intake or physical activity were further adjusted for the season when the baseline and the end-of-study questionnaires were completed. Post hoc power was calculated based on a clustered 2-sample comparison of proportions only within the subgroup not at goal at baseline. For dietary and physical activity, we obtained significant differences between the study arms. As expected, the post hoc power for either of these outcomes was more than $80 \%$. For each of the other outcomes, the post hoc power was calculated to be below $10 \%$, owing both to the small number of nonadherent participants and the small effect size observed.

Less than $5 \%$ of data was missing for such key variables as age, sex, baseline body mass index, baseline smoking status, recruitment season, and baseline perceived risk score. There were more missing data (approximately 9\%) in the family risk score. A $\chi^{2}$ anal$y$ sis of the distribution of missing data across the main outcome variables representing change found no difference in the percentage of behavioral improvement between the participants with missing information and the ones with complete information. Henceforth, we pursued a complete case analysis, ie, an analysis involving participants for whom all pertinent information was available.

\section{RESULTS}

Of the 4,248 enrolled participants, 3,786 completed the baseline survey. FHITr had an $18 \%$ recruitment rate and an $88 \%$ retention rate (from baseline to follow-up, as summarized in Figure 2). An unknown proportion of the invited patients (estimated by chart review at $\mathrm{ENH}$ to be $13 \%)^{19}$ were ineligible because of chronic diseases. As seen in Figure 1, sites that enrolled patients with an upcoming appointment had approximately double the participation rate compared with patients invited without an appointment. Overall, the mean age of participants was 50.6 years, and most were white $(91 \%)$, female $(70 \%)$, married (76\%), insured (97\%), and at a relatively high socioeconomic level. The distribution of participants by primary care specialty was family medicine $48 \%$, internal medicine $39 \%$, and obstetrics/gynecology $12 \%$. Table 1 displays the demographic characteristics of 2,364 intervention and 1,422 control group participants. After controlling for study site and practice-level clustering, the only statistically significant difference $(P=.003)$ was proportion of participants beginning the study during different seasons (defined by 4 -month periods) of the year. The distribution of familial risk of diabetes differed by study arm, with the control group exhibiting a slightly higher proportion of weak family risk $(P=.03)$. No other differences in familial risk by study arm were observed (Table 2). Among the participants using Family Healthware, 91\% viewed their risk report and messages online.

The distribution of lifestyle and screening behaviors at baseline are summarized in Table 3. Adjusting for site, age, sex, and practice clustering, there was a significantly higher percentage of participants not at goal in the intervention group with respect to aspirin use $(P<.001)$. With the exception of aspirin use, there was no statistically significant difference across the 2 study arms. Ninety percent or more of participants were already at goal for blood pressure, cholesterol, or blood glucose testing.

Table 4 summarizes the changes in preventive behaviors from baseline to follow-up for each study arm. The highlighted categories for each behavior represent the comparison groups used to determine the outcomes. The intervention group was significantly more likely than the control group to move from not at goal status to at goal for both fruit and vegetable consumption (absolute difference in proportion $=3 \%$; odds ratio $[\mathrm{OR}]=1.29 ; 95 \%$ confidence interval $[\mathrm{CI}], 1.05-1.58)$ and physical activity (absolute difference in proportion $=4 \% ; \mathrm{OR}=1.47 ; 95 \% \mathrm{CI}$, 1.08-1.98), even after adjustment (Table 5). Con-
Table 2. Risk Levels Based on Family History for the 6 Diseases by Study Arm

\begin{tabular}{|c|c|c|c|c|c|c|}
\hline \multirow[b]{2}{*}{ Risk } & \multicolumn{3}{|c|}{$\begin{array}{c}\text { Intervention Arm }(n=2,330)^{a} \\
\text { No. }(\%)\end{array}$} & \multicolumn{3}{|c|}{$\begin{array}{c}\text { Control Arm }(n=1,255)^{a} \\
\text { No. }(\%)\end{array}$} \\
\hline & Weak & Moderate & Strong & Weak & Moderate & Strong \\
\hline $\begin{array}{l}\text { Coronary heart } \\
\text { disease }\end{array}$ & $947(41)$ & $615(26)$ & $768(33)$ & $502(40)$ & $323(26)$ & $430(34)$ \\
\hline Stroke & $1,212(52)$ & $783(34)$ & $335(14)$ & $640(51)$ & $419(33)$ & $196(16)$ \\
\hline Diabetes $^{b}$ & $1,426(61)$ & $643(28)$ & $261(11)$ & $812(65)$ & $302(24)$ & 141 (11) \\
\hline Colorectal cancer & $2,015(87)$ & $263(11)$ & $52(2)$ & $1069(85)$ & $147(12)$ & $39(3)$ \\
\hline Breast cancer & $1,799(77)$ & $305(13)$ & $226(10)$ & $990(79)$ & $139(11)$ & $126(10)$ \\
\hline Ovarian cancer & 2,107 (91) & $125(5)$ & $98(4)$ & $1135(90)$ & $73(6)$ & $47(4)$ \\
\hline
\end{tabular}


trary to expectation, the study participants exposed to the message tailored to their family history were significantly less likely to move from not having a cholesterol test in the last 5 years to having it done within 5 years than were the participants exposed to the standard health message (absolute difference in proportion $=15 \% ; \mathrm{OR}=0.34$; 95\% CI, 0.17-0.67). There was no significant intervention effect on smoking cessation or blood pressure and blood glucose testing. Controlling for the number of high-risk messages, the total number of increased-risk messages received by each participant, or baseline perceived risk of each disease did not affect the results. We found no difference in outcomes between sites that enrolled patients with appointments compared with the site that invited eligible patients regardless of upcoming appointments.

Overall, older participants moved to at goal more frequently for fruit and vegetable consumption $(P<.001)$, blood glucose testing $(P=.002)$, and cholesterol testing $(P=.003)$, whereas younger participants showed a higher propensity $(P<.001)$ to move to at goal for aspirin use. Women were significantly more likely than men to move from not at goal to at goal for fruit and vegetable consumption $(P=.01)$ and aspirin use $(P=.001)$, whereas the reverse was true with respect to blood glucose testing $(P<.001)$ and smoking cessation $(P=.048)$. Nonsmokers were more likely than current smokers to move to at goal for fruit and vegetable consumption $(P=.002)$.

\section{DISCUSSION}

FHITr is the first randomized controlled trial to assess whether systematically screening for family history and tailoring prevention messages to familial risk improves any health behaviors. ${ }^{7}$ We study hypothesized that tailoring preventive recommendations for common diseases based on an individual's family history risk of each disease could increase the percentage of patients completing the recommendation. Our results support this concept for some behaviors, showing statistically significant increases in self-reported physical activity and fruit and vegetable intake for primary care patients using Family Healthware compared with those receiving a standard prevention message. Use of Family Healthware and delivery of the tailored message did not significantly increase the percentage of adults who were at goal for other health behaviors or screening compared with a standard message. Future analysis
Table 3. Lifestyle and Screening Behaviors of Participants at Baseline by Study Arm

\begin{tabular}{|c|c|c|}
\hline Characteristics & $\begin{array}{c}\text { Intervention Arm } \\
(n=2,364) \\
\text { No. }(\%)\end{array}$ & $\begin{array}{c}\text { Control Arm } \\
(n=1,422) \\
\text { No. }(\%)\end{array}$ \\
\hline \multicolumn{3}{|l|}{ Smoking } \\
\hline Current & $185(8)$ & $108(8)$ \\
\hline Former & $701(30)$ & $415(29)$ \\
\hline Never & $1,478(62)$ & $899(63)$ \\
\hline \multicolumn{3}{|l|}{ Fruit and vegetable intake } \\
\hline$<2$ servings a day & $430(18)$ & $251(18)$ \\
\hline 2-4 servings a day & $1,546(65)$ & $939(66)$ \\
\hline$\geq 5$ servings a day & $388(17)$ & $160(16)$ \\
\hline \multicolumn{3}{|l|}{ Physical activity } \\
\hline None to less than 10 min per week & $82(4)$ & $56(4)$ \\
\hline $\begin{array}{l}1-4 \text { times a week }>10 \text { min to } \\
<30 \text { min each event }\end{array}$ & $1,587(69)$ & $928(67)$ \\
\hline $\begin{array}{l}\text { 5-6 times a week at least for } \\
30 \text { min each event }\end{array}$ & $620(27)$ & $397(29)$ \\
\hline Aspirin use $\left(<3 \mathrm{~d} / \mathrm{wk}^{\mathrm{a}}\right.$ & $1,608(83)$ & $851(73)$ \\
\hline Blood pressure measured $>1$ y ago & $191(8)$ & $99(7)$ \\
\hline Cholesterol level measured $>5$ y ago & $156(7)$ & $73(5)$ \\
\hline Blood glucose level measured $>2$ y ago & $794(34)$ & $427(30)$ \\
\hline
\end{tabular}

will determine whether the effects reported here were mediated by changes in perceptions and communications as summarized in Figure 1.

Improved adherence to recommended levels of physical activity and fruit and vegetable intake after using Family Healthware was modest, but potentially influential if the changes are maintained over time, especially given the onetime intervention used to achieve this initial change. The magnitude of changes observed in daily fruit and vegetable intake ${ }^{20}$ and physical activity ${ }^{21}$ are similar to effects observed with other minimal interventions.

The family risk level was categorized as weak for most participants for every disease except coronary heart disease, as noted in Table 2 . This group of participants could have interpreted the message of no familiar risk as approval to engage in unhealthy behaviors. There was no evidence of this behavior change in Table 3, however.

There are several potential reasons why the FHITr study failed to show benefits for other health behaviors and screening. First, an unexpectedly high proportion of the study participants at baseline were already at goal for some of the recommended lifestyle and screening behaviors. As a result, study participants had very little room for improvement, thus limiting the power to detect a difference for smoking cessation, aspirin use, and blood pressure and blood glucose 
Table 4. Behavioral Change Subgroups for Each Outcome by Study Arm

\begin{tabular}{|c|c|c|c|c|c|}
\hline Behavior Change Groups ${ }^{a}$ & $\begin{array}{c}\text { Intervention } \\
\text { Arm }^{\mathrm{b}} \\
\text { No. }(\%)\end{array}$ & $\begin{array}{l}\text { Control } \\
\text { Arm } \\
\text { No. }(\%)^{b}\end{array}$ & Behavior Change Groups ${ }^{a}$ & $\begin{array}{c}\text { Intervention } \\
\text { Arm }^{\text {b }} \\
\text { No. (\%) }\end{array}$ & $\begin{array}{l}\text { Control } \\
\text { Arm } \\
\text { No. }(\%)^{\mathrm{b}}\end{array}$ \\
\hline Smoking & 2,110 & 1,278 & Aspirin use & 1,959 & 1,159 \\
\hline Maintained smoking ${ }^{c}$ & $129(6)$ & $78(6)$ & Maintained $<3 \mathrm{~d} / \mathrm{wk}^{\mathrm{c}}$ & $1,458(74)$ & $775(67)$ \\
\hline Quit smoking & $26(2)$ & $17(2)$ & Increased to $\geq 3 \mathrm{~d} / \mathrm{wk}^{\mathrm{c}}$ & $150(8)$ & $76(7)$ \\
\hline Maintained never or former & $1,944(92)$ & $1,177(92)$ & Maintained $\geq 3 \mathrm{~d} / \mathrm{wk}$ & $307(16)$ & $251(22)$ \\
\hline Started smoking & $11(1)$ & $6(1)$ & Decreased to $<3 \mathrm{~d} / \mathrm{wk}$ & $44(2)$ & $57(5)$ \\
\hline Fruit and vegetable intake & 2,111 & 1,278 & Blood pressure measured ${ }^{d}$ & 2,110 & 1,277 \\
\hline Maintained $<5$ servings a dayc & $1,560(74)$ & $973(76)$ & Still no measurement within & $16(1)$ & $7(1)$ \\
\hline Increased to $>5$ servings a dayc & $193(9)$ & $89(7)$ & past yearc & & \\
\hline Maintained $\geq 5$ servings a day & $261(12)$ & $159(12)$ & Measurement obtainedc & $147(7)$ & $78(6)$ \\
\hline Moved to $<5$ servings a day & $97(5)$ & $57(5)$ & $\begin{array}{l}\text { Measurement within } 1 \text { y for entire } \\
\text { study }\end{array}$ & $1,896(90)$ & $1,155(91)$ \\
\hline Physical activity & 2,033 & 1,236 & Measurement no longer within $1 \mathrm{y}$ & $51(2)$ & $37(3)$ \\
\hline $\begin{array}{l}\text { Maintained physical activity } \\
<5-6 \text { times a week for } \\
<30 \text { min each event }{ }^{c}\end{array}$ & $1,249(62)$ & $782(63)$ & $\begin{array}{l}\text { Cholesterol level measurede } \\
\text { Still no measurement in past } 5 y^{c}\end{array}$ & $\begin{array}{l}2,025 \\
\quad 42(2)\end{array}$ & $\begin{array}{l}1,203 \\
13(1)\end{array}$ \\
\hline Increased physical activity & $218(11)$ & $99(8)$ & Measurement obtainedc & $51(2)$ & $31(3)$ \\
\hline $\begin{array}{l}\text { 5-6 times a week for } \\
\geq 30 \text { min each event }\end{array}$ & & & $\begin{array}{l}\text { Measurement within } 5 \text { y } \\
\text { for entire study }\end{array}$ & $1,857(92)$ & $1,124(93)$ \\
\hline $\begin{array}{l}\text { Maintained 5-6 times a week } \\
\text { for } \geq 30 \text { min each event }\end{array}$ & $412(20)$ & $240(20)$ & Measurement lapsed & $75(4)$ & $35(3)$ \\
\hline \multirow{4}{*}{$\begin{array}{l}\text { Decreased physical activity } \\
<5-6 \text { times a week for } \\
<30 \text { min each event }\end{array}$} & \multirow[t]{4}{*}{$152(7)$} & \multirow[t]{4}{*}{$114(9)$} & $\begin{array}{l}\text { Blood glucose level measured } \\
\text { Still no measurement in past } 2 y^{c}\end{array}$ & $\begin{array}{l}1,726 \\
103(6)\end{array}$ & $\begin{array}{l}1,034 \\
54(5)\end{array}$ \\
\hline & & & Measurement obtained & $120(7)$ & $51(5)$ \\
\hline & & & $\begin{array}{l}\text { Measurement within } 2 \text { y } \\
\text { for entire study }\end{array}$ & $1,466(85)$ & $906(88)$ \\
\hline & & & Measurement lapsed & $37(2)$ & $23(2)$ \\
\hline
\end{tabular}

a The maintained category means the participants had the same behavior identified at baseline and month 6 based upon self-report. The increased category means the participants were not at goal at baseline and reported at goal for the specific behavior at month 6 .

${ }^{b}$ The sample size for each behavior does not equal the total study sample for each arm because of missing or incomplete data.

c Subset of the study population used to determine whether the intervention was significantly more effective in moving participants to at goal compared with the control intervention.

${ }^{\mathrm{d}}$ For blood pressure measurement, the goal was to have had a blood pressure reading by physician within the last year. The category "Still no measurement within past year" reported no blood pressure measurement within a year of baseline and at month 6 . The category "Measurement obtained" represents participants with no blood pressure measurement within a year at baseline but who reported a blood pressure measurement within a year at month 6 .

e For cholesterol, the goal was a cholesterol level measurement within the last 5 years. The category "Still no measurement in past $5 \mathrm{y}$ " means no cholesterol level was measured within 5 years from baseline to month 6 . The category "Measurement obtained" represents participants with no cholesterol level measurement within 5 years at baseline but who reported cholesterol level measurement within 5 years at month 6 .

${ }^{f}$ For blood glucose, the goal was a blood glucose level measurement within the last 2 years. The category "Still no measurement in past 2 " means no blood glucose level measured within 2 years of baseline and month 6 . The category "Measurement obtained" represents participants with no blood glucose level measurements within 2 years at baseline but who reported a blood glucose level measurement within 2 years at month 6 .

\section{Table 5. Contrast of Movement From Not at Lifestyle Goal to at Goal Compared With Persistently Not at Goal for Each Behavior by Study Arm}

\begin{tabular}{lc}
\hline Variable & OR (95\% CI) \\
\hline Quit smoking & $1.18(0.47-2.95)$ \\
Increased to $\geq 5$ serving of fruit and vegetables each day & $1.29(1.05-1.58)$ \\
Increased physical activity to $5-6$ times a week for $\geq 30$ min each event & $1.47(1.08-1.98)$ \\
Aspirin use increased to $\geq 3 \mathrm{~d} / \mathrm{wk}$ & $0.91(0.64-1.29)$ \\
Blood pressure measured by health care professional within the last year ${ }^{\mathrm{b}}$ & $1.44(0.29-7.16)$ \\
Blood cholesterol level measured within 5 y & $0.34(0.17-0.67)$ \\
Blood glucose level measured within 2 y & 1.08 (0.61-1.91) \\
\hline Cl = confidence interval; OR = odds ratio. & \\
a Odds ratio exhibits odds of the intervention group moving in a positive direction with reference to the control group. \\
Logistic regression model is adjusted for practice clustering and site, baseline body mass index, sex, baseline smoking \\
status (except for quit smoking variable), frequency of moderate/strong family risks, and risk perception score for the \\
6 diseases. Diet and physical activity variables were further adjusted for season in which questionnaires were filled out. \\
\\
b Model run without site adjustment.
\end{tabular}

testing. Although participants were active patients in primary care practices, it is unclear why the recruitment process resulted in such a high proportion of at goal participants. Recruitment materials entitled, Evaluation of Tools for Health Promotion and Disease Prevention, described the study goal to help people make decisions about health services. Patients with a commitment to disease prevention may have been more likely to participate. 
Second, the study population overrepresented white, female, married, and insured patients with a relatively high socioeconomic level compared with the populations served by the clinics. Efforts to recruit a more diverse study population failed. A barrier to recruiting a more diverse sample may have been the need for computer literacy and access. Participants, however, could also participate in the study by telephone or in person, as was done by $9 \%$ of participants. ${ }^{17}$ Although computer and Internet use is expanding rapidly in the United States, large disparities exist between ethnic and racial groups. ${ }^{22,23}$ Thus, comfort with using computers may have contributed to a biased study sample. Related studies ${ }^{24,25}$ have identified lack of access to a computer, absence of family history, and concerns about Internet security and privacy as some of the main barriers to using an Internet family history risk assessment tool.

The Family Healthware-tailored messages ranged from 8 to 15 pages. Furthermore, the reading grade level of the messages has been assessed at $11.8 .^{26}$ It is thus possible that the messages were not fully comprehended by participants. A simple 1-page generic message may have been easier to access and appeared to be more effective in convincing participants to have a blood cholesterol test. The messages delivered by Family Healthware could be shortened, highlighting key components, reducing the reading grade level, and providing more directive action steps. The messages used were standard health messages that had been used in public health settings for years. It may be time to reexamine these messages in terms of reading level, communication theory, and behavioral change theories.

We do not have data on whether the physicians reviewed the messages the study participants received or made any recommendations concerning the behaviors. Our survey of a limited physician subsample suggests they reviewed the messages for three-fourths of the participants. The degree to which physicians reinforced the messages, however, is unknown.

The study outcomes are also limited by a single (6-month) follow-up observation time. The observational interval was determined by the funding agency and resources available to conduct the study. Conclusions are also limited by the self-reported nature of the data, in that more intervention participants may have decided to endorse the socially acceptable responses once prompted by the Family Healthware reports.

This study was carried out in primary care practices because they are the locus for delivery of many preventive services. ${ }^{27} \mathrm{We}$ did not examine the impact of Family Healthware on physicians' recognition of their patients' familial disease risks or on physicianpatient interactions regarding preventive care. Medical record reviews at ENH practices did show that Family Healthware disclosed increased familial disease risks not otherwise found in a substantial proportion of participants' medical records. ${ }^{28}$ Effective ways to engage practices and physicians in recognizing familial disease risk and enacting risk-appropriate prevention strategies require further exploration. For example, electronic family history tools like Family Healthware could reduce some of the time spent collecting and processing information in the office. A key consideration will be how to integrate the information into the patient flow of the primary care practice. Simply placing a note in the electronic health record along with an electronic message to physicians outside the context of a patient visit will be insufficient to induce physicians to make familial risk-based changes to their patients' medical care. ${ }^{29}$ Importing the information and risk classification into an electronic health record's problem list with decision support built around the risk level might be more effective than the intervention evaluated here.

Data are still needed to show that familial risk data change preventive behaviors and health outcomes. Future research needs to recruit a more diverse study population, including participants not up to date on recommended screening. In addition, future research should examine how physicians use the output from tools like Family Healthware and how it affects communication between patients and physicians. Does Family Healthware provide new information to the patient or physician? Further, in the emerging context of the patient-centered medical home and models of team-based care, might such a tool help nonphysician staff to engage patients around familial risk and health behavior? Finally, it remains to be determined whether the development, deployment, and integration of tools such as Family Healthware into practice information systems will be cost effective.

Physicians have long used information about a patient's family history, lifestyle, and exposures. The modest positive impact of the FHITr intervention on dietary intake and exercise behaviors suggests that familial risk may be an important motivator of health behavior change. This study, however did not address how to improve the delivery and use of this information. FHITr has shown that technology to systematize family history assessment is feasible, but we need more insights into how it will be used and its place in preventive care.

To read or post commentaries in response to this article, see it online at http://www.annfammed.org/cgi/content/full/9/1/3.

Key words: Disease prevention; family history questionnaire; risk assessment

Submitted January 12, 2010; submitted, revised, July 19, 2010; accepted August 9, 2010. 
Funding support: The Family Healthware Impact Trial (FHITr) was supported through cooperative agreements between the CDC and the Association for Prevention Teaching and Research (ENH \#U50/CCU300860 TS-1216) and the American Association of Medical Colleges (UM \#U36/ CCU319276 MM-0789 and CWR \#U36/CCU319276 MM0630). Dr Acheson also received salary support from the National Cancer Institute K07 CA086958 in Cancer Prevention and Control.

Acknowledgements: The FIHTr Group extends our gratitude to the patients, physicians, and their office staff for participating in this study. Without their time and effort, the study would not have been possible.

The Family Healthware Impact Trial (FHITr) Group consists of the collaborators listed below:

From the CDC: Paula W. Yoon, SCD, MPH; Rodolfo Valdez, PhD; Margie Irizarry-De La Cruz, MPH; Muin J. Khoury, MD, PhD; Cynthia Jorgensen, DrPH; from the UCLA Center for Health Policy Research: Maren T. Scheuner, MD, MPH; from UM Northshore University Health System, Evanston, Illinois: Wendy S. Rubinstein, MD, PhD, Principal Investigator; Shaheen Khan, MS, MBA, MPH; from Northwestern University Feinberg School of Medicine, Chicago, Illinois: Suzanne M. O’Neill, MA, MS, PhD, Principal Investigator; Nan Rothrock, PhD; Jennifer L. Beaumont, MS; Dawood Ali, MS; from the University of Illinois at Chicago, Chicago, Illinois: Erin J. Starzyk, MPH; from Boston University School of Public Health: Catharine Wang, PhD, MSc; from the University of Michigan: Mack T. Ruffin IV, MD, MPH, Principal Investigator; Donald Nease, MD, Principal Investigator; from Case Western Reserve University, University Hospitals Case Medical Center, Cleveland, Ohio: Louise S. Acheson, MD, MS, Principal Investigator; Stephen J. Zyzanski, PhD; Georgia L. Wiesner, MD; James Werner, PhD; from the American Academy of Family Physicians' National Research Network: Wilson D. Pace, MD, Principal Investigator; James M. Galliher, PhD; Elias Brandt, BS, BA.

\section{References}

1. Yoon PW, Chen B, Faucett $A$, et al. Public health impact of genetic tests at the end of the 20th century. Genet Med. 2001;3(6):405-410

2. Khoury MJ, Gwinn M. What role for public health in genetics and vice versa? Community Genet. 2006;9(4):282, author reply 283.

3. Khoury MJ. Genetics and genomics in practice: the continuum from genetic disease to genetic information in health and disease. Genet Med. 2003;5(4):261-268.

4. Williams RR, Hunt SC, Heiss G, et al. Usefulness of cardiovascular family history data for population-based preventive medicine and medical research (the Health Family Tree Study and the NHLBI Family Heart Study). Am J Cardiol. 2001;87(2):129-135.

5. Acheson LS, Wiesner GL, Zyzanski SJ, Goodwin MA, Stange KC. Family history-taking in community family practice: implications for genetic screening. Genet Med. 2000;2(3):180-185.

6. Berg AO, Baird MA, Botkin JR, et al. National Institutes of Health State-of-the-Science Conference Statement: Family History and Improving Health. Ann Intern Med. 2009;151(12):872-877.

7. Wilson BJ, Qureshi N, Santaguida P, et al. Systematic review: family history in risk assessment for common diseases. Ann Intern Med. 2009;151(12):878-885.

8. Rogers J, Durkin M. The semi-structured genogram interview: I. Protocol, II. Evaluation. Fam Syst Med. 1984;2:176-188.

9. Waters I, Watson W, Wetzel W. Genograms. Practical tools for family physicians. Can Fam Physician. 1994;40:282-287.

10. Rich EC, Burke W, Heaton CJ, et al. Reconsidering the family history in primary care. J Gen Intern Med. 2004;19(3):273-280.
11. Qureshi N, Wilson B, Santaguida P, et al. Collection and use of cancer family history in primary care. Evid Rep Technol Assess (Full Rep). 2007;(159):1-84

12. Yoon PW, Scheuner MT, Khoury MJ. Research priorities for evaluating family history in the prevention of common chronic diseases. Am J Prev Med. 2003;24(2):128-135.

13. Scheuner MT, Wang SJ, Raffel LJ, Larabell SK, Rotter JI. Family history: a comprehensive genetic risk assessment method for the chronic conditions of adulthood. Am J Med Genet. 1997;71(3):315-324.

14. Emery J, Morris H, Goodchild R, et al. The GRAIDS Trial: a cluster randomised controlled trial of computer decision support for the management of familial cancer risk in primary care. $\mathrm{Br} J$ Cancer. 2007;97(4):486-493.

15. Yoon PW, Scheuner MT, Jorgensen C, Khoury MJ. Developing Family Healthware, a family history screening tool to prevent common chronic diseases. Prev Chronic Dis. 2009;6(1):A33.

16. Facio FM, Feero WG, Linn A, Oden N, Manickam K, Biesecker LG. Validation of My Family Health Portrait for six common heritable conditions. Genet Med. 2010;12(6):370-375.

17. O'Neill SM, Rubinstein WS, Wang C, et al.; Family Healthware Impact Trial group. Familial risk for common diseases in primary care: the Family Healthware Impact Trial. Am J Prev Med. 2009;36(6):506-514.

18. Ma Y, Olendzki BC, Li W, et al. Seasonal variation in food intake, physical activity, and body weight in a predominantly overweight population. Eur J Clin Nutr. 2006;60(4):519-528.

19. Acheson LS, Wang C, Zyzanski SJ, et al.; Family Healthware Impact Trial (FHITr) Group. Family history and perceptions about risk and prevention for chronic diseases in primary care: a report from the family healthware impact trial. Genet Med. 2010;12(4):212-218.

20. Pomerleau J, Lock K, Knai C, McKee M. Interventions designed to increase adult fruit and vegetable intake can be effective: a systematic review of the literature. J Nutr. 2005;135(10):2486-2495.

21. Hillsdon M, Foster C, Thorogood M. Interventions for promoting physical activity. Cochrane Database Syst Rev. 2005;(1):CD003180.

22. Kim G. Three out of four Americans have access to the Internet. Nielsen//Net Ratings. March 18, 2004. http://www.nielsen-online. com/pr/pr_040318.pdf. Accessed Sep 21, 2005.

23. Cole J, Suman M, Shcramm P, et al. The Digital Future Report: Surveying the Digital Future, Year Four. Los Angeles, CA: Center for the Digital Future, USC Annenberg School for Communication; 2004.

24. Simon C, Acheson L, Burant C, et al. Patient interest in recording family histories of cancer via the Internet. Genet Med. 2008;10(12): 895-902.

25. Acheson L, Wiesner G, Lynn A. Acceptability of an internet tool for familial cancer risk assessment among women having mammograms. Paper presented at: American College of Medical Genetics Annual Meeting, 2010; Albuquerque, NM.

26. Wang C, Gallo RE, Fleisher L, et al. Literacy assessment of family health history tools for Public Health prevention. Public Health Genomics. 2010 Jan 4. [Epub ahead of print].

27. Stange KC, Flocke SA, Goodwin MA, Kelly RB, Zyzanski SJ. Direct observation of rates of preventive service delivery in community family practice. Prev Med. 2000;31(2 Pt 1):167-176.

28. O’Neill S, Starzyk E, Kattezham R, et al. Comparison of Family Healthware and Physicians' Family History Documentation among 1124 Patients. American Society of Human Genetics. Philadelphia, PA; 2008.

29. Volk LA, Staroselsky M, Newmark LP, et al. Do physicians take action on high risk family history information provided by patients outside of a clinic visit? Stud Health Technol Inform. 2007;129(Pt 1):13-17. 